\title{
ON FUNCTIONS DERIVED FROM REGULARLY VARYING FUNCTIONS
}

\author{
LAURENS DE HAAN*
}

(Received 9 October 1975; revised 8 March 1976)

\section{Introduction and notation}

A generalization of Karamata's theorem on integrals of regularly varying functions is proved. Using Laplace-Stieltjes transforms it is shown that any regularly varying function with exponent $\alpha(\alpha+1 \notin \mathbf{N})$ is asymptotic to another regularly varying function all of whose derivations are regularly varying.

Suppose $U$ is a positive function on $\mathbf{R}^{+} . U$ is regularly varying at $\infty$ (or $0+$ ) with exponent $\alpha$, in short $\alpha$-varying, notation $U \in R V_{\alpha}^{(\infty)}$ (or $R V_{\alpha}^{(0)}$ respectively), if for all $x>0$

$$
\frac{U(t x)}{U(t)} \rightarrow x^{\alpha}
$$

as $t \rightarrow \infty$ (or $t \downarrow 0$ respectively); cf. Karamata (1930) and (1933), Feller (1971) chapter VIII, 8 and XIII, 5 .

If $U$ is non-decreasing and if for suitable functions $a(t)>0$ and $b(t)$ and all $x>0$

$$
\frac{U(t x)-b(t)}{a(t)} \rightarrow \log x
$$

as $t \rightarrow \infty$ we say $U \in \Pi^{(x)}$. If $U$ is non-increasing and if for suitable functions $a(t)>0$ and $b(t)$ and all $x>0$

$$
\frac{U(t x)-b(t)}{a(t)} \rightarrow \log x
$$

as $t \downarrow 0$ we say $U \in \Pi^{(0)}$; cf. de Haan (1970), section I, 4.

* Research supported in part by the Netherlands Organisation for the Advancement of Pure Research (Z.W.O.) and in part by CSIRO, Division of Mathematics and Statistics. 


\section{Integrals of regularly varying functions}

We start from a well known result. Suppose $F$ is a probability distribution function and $F(0+)=0$. Then (Feller (1971), VIII, 9 th. 2. cf. Pitman (1968), lemma 3) for $\alpha>0, \beta<0, \alpha+\beta>0$

(P1)

$$
\begin{aligned}
& \int_{0}^{x} t^{\alpha} d F(t) \in R V_{\alpha+\beta}^{(\infty)} \Leftrightarrow \int_{0}^{x} t^{\alpha-1}(1-F(t)) d t \in R V_{\alpha+\beta}^{(\alpha)} \\
& \Leftrightarrow 1-F(x) \in R V_{\beta}^{(\infty)} \Leftrightarrow \lim _{x \rightarrow \infty} \frac{\int_{0}^{x} t^{\alpha-1}(1-F(t)) d t}{x^{\alpha}(1-F(x))}=\frac{1}{\alpha+\beta} \\
& \Leftrightarrow \lim _{x \rightarrow \infty} \frac{\int_{0}^{x} t^{\alpha} d F(t)}{x^{\alpha}(1-F(x))}=\frac{-\beta}{\alpha+\beta} .
\end{aligned}
$$

A variant is the following. Suppose $U$ is non-decreasing, $U(0+)=0$, then for $\alpha>0, \beta>0$

$$
\begin{aligned}
& \int_{0}^{x} t^{\alpha} d U(t) \in R V_{\alpha+\beta}^{(\infty)} \Leftrightarrow \int_{0}^{x} t^{\alpha-1} U(t) d t \in R V_{\alpha+\beta}^{(\infty)} \\
& \Leftrightarrow U(x) \in R V_{\beta}^{(\infty)} \quad \Leftrightarrow \lim _{x \rightarrow \infty} \frac{\int_{0}^{x} t^{\alpha-1} U(t) d t}{x^{\alpha} U(x)}=\frac{1}{\alpha+\beta} \\
& \Leftrightarrow \lim _{x \rightarrow \infty} \frac{\int_{0}^{x} t^{\alpha} d U(t)}{x^{\alpha} U(x)}=\frac{\beta}{\alpha+\beta} .
\end{aligned}
$$

We want to present some analogous statements. Proofs are given in the next section. Firstly for probability distributions $F$ and $\alpha>0$

$$
\begin{aligned}
& \int_{0}^{x} t^{\alpha} d F(t) \in R V_{0}^{(x)} \Leftrightarrow \int_{0}^{x} t^{\alpha-1}(1-F(t)) d t \in R V_{0}^{(x)} \\
& \Leftrightarrow \lim _{x \rightarrow \infty} \frac{\int_{0}^{x} t^{\alpha-1}(1-F(t)) d t}{x^{\alpha}(1-F(x))}=\infty \Leftrightarrow \lim _{x \rightarrow \infty} \frac{\int_{0}^{x} t^{\alpha} d F(t)}{x^{\alpha}(1-F(x))}=\infty .
\end{aligned}
$$

This is the case $\alpha+\beta=0$ of (P1). A sufficient (but not necessary) condition is $1-F(x) \in R V_{-\alpha}^{(x)}$.

Next suppose $U$ is as above. For $\alpha>0$

$$
U \in \Pi^{(\infty)} \Leftrightarrow \int_{0}^{x} t^{\alpha} d U(t) \in R V_{\alpha}^{(\infty)} \Leftrightarrow \int_{x}^{\infty} t^{-\alpha} d U(t) \in R V_{-\alpha}^{(\infty)} .
$$

This is the case $\beta=0$ of (P2). 
Finally suppose $U_{1}$ is non-decreasing, $U_{2}$ is continuous and strictly increasing, $U_{2}(0+)=0$. Suppose $\alpha>0, \beta>0$.

(P5) Any two of the following statements imply the others.
a. $U_{1} \in R V_{\alpha}^{(x)}$
b. $U_{2} \in R V_{\beta}^{(x)}$
c. $\int_{0}^{x} U_{1}(t) d U_{2}(t) \in R V_{\alpha+\beta}^{(\infty)}$
d. $\lim _{x \rightarrow \infty} \frac{\int_{0}^{x} U_{1}(t) d U_{2}(t)}{U_{1}(x) U_{2}(x)}=\frac{\beta}{\alpha+\beta}$

This generalizes (P2). Similarly for functions in $\Pi^{(\infty)}$ we have the following.

(P6) Suppose $U_{1} \in R V_{\alpha}^{(\infty)}(\alpha>0), U_{1}$ is continuous and strictly increasing, $U_{1}(0+)=0$.

$$
U_{2}(x) \in \Pi^{(x)} \Leftrightarrow \int_{0}^{x} U_{1}(t) d U_{2}(t) \in R V_{\alpha}^{(\infty)} \Leftrightarrow \int_{x}^{\infty} \frac{d U_{2}(t)}{U_{1}(t)} \in R V_{-\alpha .}^{(\infty)}
$$

This generalizes (P4).

Remark. Property P5 may be used to generalize a result on convergence of moments for sample extremes, see Pickands (1968).

\section{Proofs and remarks}

Proof OF (P3).

$$
\int_{0}^{x} t^{\alpha} d F(t) \in R V_{0}^{(\infty)}
$$

if and only if

$$
\lim _{x \rightarrow \infty} \int_{0}^{x} t^{\alpha} d F(t) /\left\{x^{\alpha}(1-F(x))\right\}=\infty
$$

by Feller (1971), VIII, 9 th. 2 (part iii). Now

$$
\begin{aligned}
& \lim _{x \rightarrow \infty} \int_{0}^{x} t^{\alpha} d F(t) /\left\{x^{\alpha}(1-F(x))\right\}=\infty \\
& \Leftrightarrow \lim _{x \rightarrow \infty} \int_{0}^{x} t^{\alpha-1}(1-F(t)) d t /\left\{x^{\alpha}(1-F(x))\right\}=\infty
\end{aligned}
$$

is a matter of partial integration. If 


$$
\alpha(x)=\int_{0}^{x} t^{\alpha-1}(1-F(t)) d t /\left\{x^{\alpha}(1-F(x))\right\} \rightarrow \infty \quad(x \rightarrow \infty)
$$

then

$$
\int_{0}^{x} t^{\alpha-1}(1-F(t)) d t=\left\{\int_{0}^{1} t^{\alpha-1}(1-F(t)) d t\right\} \exp \int_{1}^{x}\{t \alpha(t)\}^{-1} d t
$$

and the latter is in $R V_{0}^{(\infty)}$ by the representation theorem for regularly varying functions. If $\int_{0}^{x} t^{\alpha-1}(1-F(t)) d t \in R V_{0}^{(x)}$ then by an obvious extension of the argument in Feller (1971, prop. 8 p. 22)

$$
\lim _{x \rightarrow \infty} \int_{0}^{x} t^{\alpha-1}(1-F(t)) d t /\left\{x^{\alpha}(1-F(x))\right\}=\infty .
$$

Remark. The statements of (P3) with $\alpha=1$ are the necessary and sufficient conditions for a weak law of large numbers for positive random variables (Feller (1971), VII, 7 th. 2).

Remark. The statements of (P3) are implied (Feller (1971), VIII, 9 th. 2) by the set of equivalent statements $(\alpha>0, \beta>\alpha-1)$

$$
\begin{aligned}
& 1-F(x) \in R V_{-\alpha}^{(x)} \Leftrightarrow \int_{0}^{x} t^{\alpha-1}(1-F(t)) d t \in \Pi^{(\infty)} \\
& \Leftrightarrow \int_{0}^{x} t^{\alpha} d F(t) \in \Pi^{(\alpha)} \Leftrightarrow \int_{0}^{x} t^{\beta}(1-F(t)) d t \in R V_{\beta-\alpha+1}^{(\infty)}
\end{aligned}
$$

(the equivalence of these statements follows from (P4)).

ProOf OF (P5). $U_{2}$ has a proper inverse $U_{2}^{-1}$. So

$$
\int_{0}^{x} U_{1}(t) d U_{2}(t)=\int_{0}^{U_{2}(x)} U_{1}\left(U_{2}^{-1}(s)\right) d s .
$$

We shall write $U_{1} \circ U_{2}^{-1}(s)$ for the compound function $U_{1}\left(U_{2}^{-1}(s)\right)$.

Assume a) and b). Then $U_{1} \circ U_{2}^{-1} \in R V_{\alpha / \beta}^{(x)}$, and hence

$$
\begin{aligned}
\lim _{y \rightarrow \infty} \frac{\int_{0}^{y} U_{1}(t) d U_{2}(t)}{U_{1}(y) U_{2}(y)} & =\lim _{x \rightarrow \infty} \frac{\int_{0}^{U_{2}^{-1}(x)} U_{1}(t) d U_{2}(t)}{x U_{1} \circ U_{2}^{-1}(x)} \\
& =\lim _{x \rightarrow \infty} \frac{\int_{0}^{x} U_{1} \circ U_{2}^{-1}(s) d s}{x U_{1} \circ U_{2}^{-1}(x)}=\frac{\beta}{\alpha+\beta} .
\end{aligned}
$$

Assume b) and c). The compound function

$$
\int_{0}^{U_{2}^{-1}(x)} U_{1}(t) d U_{2}(t)=\int_{0}^{x} U_{1} \circ U_{2}^{-1}(s) d s
$$


then belongs to $R V_{\beta}^{(x)_{1}(\alpha+\beta)}$. Since $U_{1} \circ U_{2}^{-1}$ is monotone, it follows $U_{1} \circ U_{2}^{-1} \in$ $R V_{\beta^{-1}{ }_{\alpha}}^{(x)}$. Hence

$$
U_{1}=U_{1} \circ U_{2}^{-1} \circ U_{2} \in R V_{\alpha}^{(\infty)} .
$$

Assume a) and c). It is well known that there is a continuous and strictly increasing function $U_{3}$ such that $U_{3}(0+)=0$ and $U_{1}(x) \sim U_{3}(x)$ as $x \rightarrow \infty$. Then $\int_{0}^{x} U_{3}(t) d U_{2}(t) \sim \int_{0}^{x} U_{1}(t) d U_{2}(t)$ as $x \rightarrow \infty$. The compound function

$$
\int_{0}^{U_{3}^{-1}(x)} U_{3}(t) d U_{2}(t)=\int_{0}^{x} s d U_{2} \circ U_{3}^{-1}(s)
$$

is in $R V_{\alpha{ }^{\prime}(\alpha+\beta)}^{(x)_{1}}$. By (P2) then $U_{2} \circ U_{3}^{-1} \in R V_{\alpha}^{(x)}{ }_{1}$. Hence $U_{2}=$ $U_{2} \circ U_{3}^{-1} \circ U_{3} \in R V_{\beta}^{(x)}$.

Assume d) then

$$
\begin{aligned}
\lim _{x \rightarrow \infty} \frac{\int_{0}^{x} U_{1} \circ U_{2}^{-1}(s) d s}{x U_{1} \circ U_{2}^{-1}(x)} & =\lim _{x \rightarrow \infty} \frac{\int_{0}^{U_{2}(x)} U_{1} \circ U_{2}^{-1}(s) d s}{U_{2}(x) U_{1} \circ U_{2}^{-1}\left(U_{2}(x)\right)} \\
& =\lim _{x \rightarrow \infty} \frac{\int_{0}^{x} U_{1}(t) d U_{2}(t)}{U_{1}(x) U_{2}(x)}=\frac{\beta}{\alpha+\beta} .
\end{aligned}
$$

Hence $U_{1} \circ U_{2}^{-1} \in R V_{\beta}^{(x){ }_{1}}$. Once we know this a) and b) are equivalent. If in addition to d) we assume c) then $U_{1}(x) U_{2}(x)=U_{2}(x) U_{1} \circ U_{2}^{-1}\left(U_{2}(x)\right)=$ $U_{+} \circ U_{2}(x) \in R V_{\alpha+\beta}^{(x)}$ where $U_{4}(x)=x U_{1} \circ U_{2}^{-1}(x)$. Clearly $U_{4} \in R V_{\beta}^{(x) l_{(\alpha+\beta)}}$ hence $U_{2} \in R V_{\beta}^{(\infty)}$.

The implications abd $\Rightarrow c$ and bcd $\Rightarrow$ a are trivial. Suppose abc then from $U_{1} \circ U_{2}^{-1} \in R V_{\beta^{-1} \alpha}$ it follows as above

$$
\lim _{x \rightarrow \infty} \frac{\int_{0}^{x} U_{1}(t) d U_{2}(t)}{U_{1}(x) U_{2}(x)}=\lim _{x \rightarrow \infty} \frac{\int_{0}^{x} U_{1} \circ U_{2}^{-1}(s) d s}{x U_{1} \circ U_{2}^{-1}(x)}=\frac{\beta}{\alpha+\beta} .
$$

ProOf of (P6).

$$
\begin{aligned}
& U_{2} \in \Pi^{(x)} \Leftrightarrow U_{2} \circ U_{1}^{-1} \in \Pi^{(x)} \Leftrightarrow \int_{0}^{x} t d U_{2} \circ U_{1}^{-1}(t) \in R V_{1}^{(x)} \\
& \Leftrightarrow \int_{0}^{x} U_{1}(t) d U_{2}(t)=\int_{0}^{U_{1}(x)} t d U_{2} \circ U_{1}^{-1}(t) \in R V_{\alpha}^{(x)}
\end{aligned}
$$

and similarly for the third statement of (P6). The second equivalence above follows from de Haan (1970), theorem 1.4.1.b.

As to the first equivalence: suppose $U_{2} \in \Pi^{(x)}$ and $U_{1}^{-1} \in R V_{\alpha}^{(x)}$, then for all $x>0$ 


$$
\lim _{r \rightarrow \infty} \frac{U_{2}\left(\frac{U_{1}^{-1}(t x)}{U_{1}^{-1}(t)} \cdot U_{1}^{-1}(t)\right)-U_{2}\left(U_{1}^{-1}(t)\right)}{U_{2}\left(\frac{U_{1}^{-1}(t e)}{U_{1}^{-1}(t)} \cdot U_{1}^{-1}(t)\right)-U_{2}\left(U_{1}^{1}(t)\right)}=\frac{\log x^{\alpha^{-1}}}{\log e^{\alpha^{-1}}}=\log x .
$$

For the converse implication write $U_{2}=U_{2} \circ U_{1}^{-1} \circ U_{1}$.

\section{Derivatives}

We prove the following:

THEOREM 1. Any $\alpha$-varying function $U$ with $\alpha+1 \notin \mathbf{N}$ is asymptotic to a function $U_{1}$ with the property that the absolute values of all its derivatives are regularly varying.

Proof. First let $\alpha<0$. There is a decreasing function $U_{2}$ such that $U(x) \sim U_{2}(x)$ as $x \rightarrow \infty$. Define $U_{3}(x)=U_{2}\left(\frac{1}{x}\right)$ then $U_{3} \in R V_{-\alpha}^{(0)}$. Denote its Laplace-Stieltjes transform by $\check{U}_{3}$. Then $U_{3}(x) \sim\{\Gamma(1-\alpha)\}^{-1} \check{U}_{3}\left(\frac{1}{x}\right)$ as $x \downarrow 0$. So $U(x) \sim\{\Gamma(1-\alpha)\}^{-1} \check{U}_{3}(x)$ as $x \rightarrow x$ and latter function satisfies the requirements (property 8 p. 22 de Haan. (1970)).

Next let $\alpha>0(\alpha \notin \mathbf{N})$. There is an increasing function $U_{2}$ such that $U_{2}(0+)=0$ and $U(x) \sim U_{2}(x)$ as $x \rightarrow x$. Denote its Laplace-Stieltjes transform by $\check{U}_{2}$. Then

$$
U(x) \sim U_{2}(x) \sim \frac{1}{\Gamma(1+\alpha)} \check{U}_{2}\left(\frac{1}{x}\right) \quad \text { as } \quad x \rightarrow \infty .
$$

We shall prove that $U_{1}(x)=(\Gamma(1+\alpha))^{-1} \breve{U}_{2}\left(\frac{1}{x}\right)$ satisfies the requirements.

We have (Abramowitz and Stegun (1970) Ch. 24, 1.2.I.c.)

$$
\frac{d^{n}}{d x^{n}} \check{U}_{2}\left(\frac{1}{x}\right)=\sum_{m=1}^{n} \frac{n !}{m !}\left(\begin{array}{c}
n-1 \\
m-1
\end{array}\right)(-1)^{n} x^{-n-m} \check{U}_{2}^{(m)}\left(\frac{1}{x}\right) .
$$

By property 8 of de $\operatorname{Haan}(1970)$, p. 22) for $m=1,2, \cdots$

$$
x^{-m} \check{U}_{2}^{(m)}\left(\frac{1}{x}\right) \sim(-\alpha)(-\alpha-1) \cdots(-\alpha-m+1) \check{U}_{2}\left(\frac{1}{x}\right)
$$

as $x \rightarrow \infty$. Hence as $x \rightarrow \infty$

$$
\frac{d^{n}}{d x^{n}} \check{U}_{2}\left(\frac{1}{x}\right) \sim n !(-1)^{n} x^{-n} \check{U}_{2}\left(\frac{1}{x}\right) \sum_{m=1}^{n}\left(\begin{array}{c}
n-1 \\
n-m
\end{array}\right)\left(\begin{array}{c}
-\alpha \\
m
\end{array}\right)
$$




$$
\begin{aligned}
& =\left(\begin{array}{c}
-\alpha+n-1 \\
n
\end{array}\right) n !(-1)^{n} x^{-n} \check{U}_{2}\left(\frac{1}{x}\right) \\
& =\alpha(\alpha-1) \cdots(\alpha-n+1) x^{-n} \breve{U}_{2}\left(\frac{1}{x}\right) .
\end{aligned}
$$

Remark. A. A. Balkema has given another proof of this result using the convolution of the function with a probability distribution.

The theorem says that within each equivalence class of asymptotically equivalent regularly varying functions there is at least one function satisfying the requirements. A similar statement with a different definition of the equivalence class holds for functions in $\Pi$. This is the analogue of the previous theorem for $\alpha=0$.

TheOREM 2. Any function $U \in \Pi^{(\infty)}$, i.e. any non-decreasing function satisfying

$$
\lim _{t \rightarrow \infty} \frac{U(t x)-b(t)}{a(t)}=\log x
$$

for all $x>0$ and suitably chosen functions $a(t)>0$ and $b(t)$ has a companion function $U_{1}$ such that $(-1)^{n+1} U_{1}^{(n)}(x) \in R V_{-n}^{(\infty)}$ for $n=1,2, \cdots$ and

$$
\lim _{t \rightarrow \infty} \frac{U(t)-U_{1}(t)}{a(t)}=0 .
$$

Proof. The Laplace-Stieltjes transform $\check{U}(t)$ of $U$ exists for all $t>0$. We shall prove that $U_{1}(x)=\check{U}\left(x^{-1} e^{-\gamma}\right)$ satisfies the requirements; here $\gamma$ is Euler's constant. By de Haan (1976) $\check{U} \in \Pi^{(0)}$ and

$$
\lim _{t \rightarrow \infty} \frac{U(t)-\check{U}\left(\frac{1}{t}\right)}{a(t)}=\gamma .
$$

As in the previous proof we have

$$
\frac{d^{n}}{d x^{n}} \check{U}\left(\frac{1}{x}\right)=\sum_{m=1}^{n} \frac{n !}{m !}\left(\begin{array}{c}
n-1 \\
m-1
\end{array}\right)(-1)^{n} x^{-n-m} \check{U}^{(m)}\left(\frac{1}{x}\right) .
$$

By the lemma in de Haan (1976) we have for the derivative $-\check{U}^{(1)}(1 / x) \in$ $R V_{-1}^{(0)}$ and by property 8 , p. 22 of de Haan (1970) for $m=1,2, \cdots$ as $x \rightarrow \infty$

$$
x^{-m} \check{U}^{(m)}\left(\frac{1}{x}\right) \sim(-1)^{m+1}(m-1) ! x^{-1} \check{U}^{(1)}\left(\frac{1}{x}\right)
$$

Hence as $x \rightarrow \infty$ 


$$
\begin{aligned}
\frac{d^{n}}{d x^{n}} & \breve{U}\left(\frac{1}{x}\right) \sim(-1)^{n} x^{-n-1} \breve{U}^{(1)}\left(\frac{1}{x}\right) \sum_{m=1}^{n}\left(\begin{array}{c}
n-1 \\
m-1
\end{array}\right) \frac{(m-1) ! n !}{m !}(-1)^{m+1} \\
& =(-1)^{n} x^{-n-1}(n-1) ! \check{U}^{(1)}\left(\frac{1}{x}\right) .
\end{aligned}
$$

\section{References}

M. Abramowitz, and I. A. Stegun, ed. (1970), Handbook of mathematical functions (National burcau of standards. Washington).

W. Feller (1971), Introduction to Probability Theory and Its Applications, Vol. 2 (Wiley, New York).

L. de Haan, (1970), 'On regular variation and its application to the weak convergence of sample extremes', Mathematisch Centrum, Amsterdam.

L. de Haan (1976), 'An Abel-Tauber theorem for Laplace transforms', J. London Math. Soc. (2), 13, $537-542$.

J. Karamata, (1930), 'Sur un mode de croissance régulière des fonctions', Mathematica (Cluj) 4, 38-53.

J. Karamata, (1933), 'Sur un mode de croissance régulière. Théorèmes fondamentaux'. Bull. Soc. Math. France 61, 55-62.

J. Pickands, (1968), 'Moment convergence of sample extremes', Ann. Math. Statist. 39, 881-889.

E. J. G. Pitman, (1968), 'On the behaviour of the characteristic function of a probability distribution in the neighbourhood of the origin', I. Austral. Math. Soc. 8, 423-443.

Econometric Institute,

Netherlands School of Economics,

Burgemeester Oudlaan 50,

Rotterdam 3016,

Netherlands. 